\title{
On Modelling Approaches for Receding-Horizon Control Design Applied to Large-Scale Sewage Systems
}

\author{
Carlos Ocampo-Martinez and Vicenç Puig
}

\begin{abstract}
In this paper, receding horizon control of largescale sewage systems is addressed considering different modelling approaches, which include several inherent continuous/discrete phenomena (overflows in sewers and tanks) and elements (weirs) in the system. This fact results in distinct behaviours depending on the dynamic state (flow/volume) of the network. These behaviours can not be neglected nor can be modelled by a pure linear representation. In order to take into account these phenomena and elements for the design of the control strategy, a modelling approach based on piece-wise linear functions (PWLF) is proposed and compared against a hybrid modelling approach previously reported by the authors. Control performance results and associated computation times considering both modelling approaches are compared by using a real case study based on the Barcelona sewer network. Results have shown an important reduction in the computation time when the PWLF-based model is used, with an acceptable suboptimality level in the closed-loop system performance.
\end{abstract}

\section{INTRODUCTION}

$\mathbf{S}$ EWER networks are complex large-scale systems since they are geographically distributed and interconnected with a hierarchical structure. Each subsystem is itself composed of a large number of elements with time-varying behaviour, exhibiting numerous operating modes and subject to dynamic changes due to external conditions (weather) and operational constraints. Most cities around the world have sewage systems that combine sanitary and storm water flows within the same network. This is why these networks are known as Combined Sewage Systems (CSS). During rain storms, wastewater flows can easily overload these CSS, thereby causing operators to dump the excess of water into the nearest receiver environment (rivers, streams or sea). This discharge to the environment, known as Combined Sewage Overflow (CSO), contains biological and chemical contaminants creating a major environmental and public health hazard. A possible solution to the CSO problem would be to enhance the current sewer infrastructure by increasing the capacity of the wastewater treatment plants (WWTP) and by building new underground retention tanks. But in order to take profit of these expensive infrastructures, it is also necessary a highly sophisticated real-time control

This work has been supported by the CICYT Ref. DPI2006-11944 of the Spanish Science and Technology Ministry, the Juan de la Cierva Research Programme (Ref. JCI-2008-2438), and the DGR of Generalitat de Catalunya (SAC group Ref. 2009/SGR/1491).

C. Ocampo-Martinez and V. Puig are with Institut de Robòtica i Informàtica Industrial, CSIC-UPC, Llorens i Artigas, 4-6, 08028 Barcelona, Spain. cocampodiri.upc.edu

V. Puig is also with the Automatic Control Department, Technical University of Catalonia (UPC), Rambla de Sant Nebridi, 10, 08222 Terrassa, Spain. vicenc.puig@upc.edu
(RTC) scheme, which ensures that high performance can be achieved and maintained under adverse meteorological conditions. The advantage of RTC applied to sewer networks has been demonstrated by an important number of researchers during the last decades, see, e.g., [1], [2].

A RTC scheme in sewage systems might be local (when flow regulation devices use only measurements taken at their specific locations) or global (when control actions are computed taking into account real-time measurements all through the network). Since a sewer network shows strong cross-relation between its elements, a global RTC is the proper strategy to manage and control this type of systems. The multivariable and large-scale nature of sewer networks have lead to the use of some variants of Receding Horizon Control (RHC) - also referred as Model Predictive Control (MPC) - as global control strategies [3], [4]. In order to use RHC within a global RTC scheme of a sewage system, a model able to predict its future states over a prediction horizon taking into account a rain forecast is needed.

Sewer networks are systems with complex dynamics since water flows through sewers in open channel. When developing a control-oriented model, there is always a tradeoff between model description accuracy and computational complexity. Several control-oriented modelling techniques presented in the literature deal with the global RTC of sewage systems, see [4], [5]. In [6], [7], a conceptual linear model based on assuming that a set of sewers in a catchment can be considered as a virtual tank is used. The main reason to use a linear model is to preserve the convexity of the optimization problems related to the RHC strategy [3]. However, there exist several inherent phenomena (overflows in sewers and tanks) and elements (weirs) in the system that result in distinct behaviour depending on the state (flow/volume) of the network. These discontinuous behaviours can not be neglected nor can be modelled by a pure linear model. Additionally, the presence of intense precipitation causes that new flow paths appear. Thus, some flow paths are not always present in the sewer network and depend on its state and disturbances (rain). The description and analysis of these continuous/discrete dynamic behaviours present in sewer networks have been previously reported by the authors [8]. In that work, an hybrid modelling approach based on the Mixed Logical Dynamical (MLD) form -introduced in [9] - oriented to the design of RHC-based RTC scheme for large-scale sewage systems was proposed. However, it was shown that the inclusion of those discontinuous behaviours in the RHC problem increases the computation time of the control law. So, some relaxation in the modelling approach 
should be thought such that it can be used in the RTC of sewer networks.

In this line, the aim of this paper is to propose an alternative modelling approach that represents the sewage system by using piece-wise linear functions (in the sequel called $P W L F$ based model or simply PWLF model), following the ideas proposed by Schechter [10]. The purpose of this modelling approach is to reduce the complexity of the RHC problem by avoiding the logical variables introduced by the MLD system representation. The idea behind the PWLF-based modelling approach consists in having a description of the network using functions that, despite their discontinuous nature, are considered as quasi-convex [11], fact that might yield to the quasi-convexity of optimization problems associated to the non-linear MPC strategy used for RTC of the sewage system [7]. In this way, the resultant optimization problems does not include integer variables, what allows saving computation time.

The remainder of the paper is organized as follows. In Section II, control-oriented modelling of sewer networks is reviewed and the proposed PWLF-based modelling approach is presented. RHC-based RTC scheme for sewage systems is briefly addressed in Section III. Section IV presents a real case study based on the Barcelona sewer network. This case study is used to compare the closed-loop performance when implementing a predictive controller based on the modelling approach in Section III and the one suggested in [8]. Section $\mathrm{V}$ shows and discusses the performance results of the comparison between the PWLF and hybrid models. Finally, main conclusions close the paper in Section VI.

\section{PWLF-based Modelling for Sewer Networks RTC}

As discussed in the introduction, sewer networks present several inherent hybrid behaviors that can not be modelled using a pure linear model. In this paper, the modelling framework based on PWLFs is used to model such behaviors. More precisely, the proposed PWLF-based modelling methodology consists in using continuous and monotonic functions to represent expressions that contain logical conditions, which describe the weirs behaviour and overflow capability of reservoirs, respectively. Indeed, these phenomena involve the switching and discontinuous behaviours of the sewage system. The PWLF approach is though as an alternative to the use of a pure hybrid modelling approach, already proposed for the RTC of sewer networks [8].

The PWLFs used to model the discontinuous behaviours of sewer networks are defined as saturation of a variable $x$ in a value $M$ (i.e., $\operatorname{sat}(x, m)$ ), and dead zone of the same variable $x$ starting in a value $M$ (i.e., $d z n(x, M)$ ). Those functions are monotonic and continuous and might lead to a quasi-convex optimization problem when formulating the MPC problem. According to [11], the global optimal solution of quasi-convex optimization problems can be obtained by using a bisection method, which is logarithmic in time. This represents an advantage with respect to the resultant mixedinteger linear problems when using a pure hybrid approach based on MLD or PWA approaches. This type of models induces an exponential complexity given by the handling of Boolean variables and the discrete optimization required.

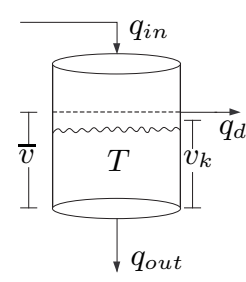

(a) Virtual tank

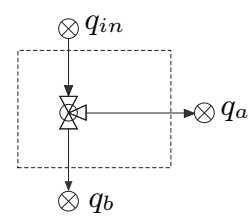

(c) Redirection gate

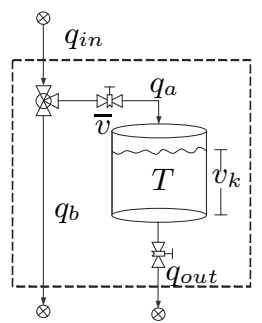

(b) Real tank

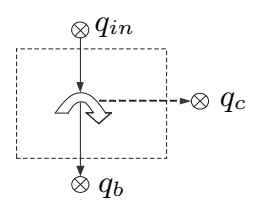

(d) Sewer pipe or weir with single inflow
Fig. 1. Conceptual schemes for sewer networks constitutive elements.

\section{A. Virtual and Real Tanks}

These elements are used as storage devices. In the case of virtual tanks (see Figure 1(a)), the mass balance of the stored volume, the tank inflows and outflows and the input rain intensity can be written as the difference equation with a sampling time $\Delta t$

$$
v_{i k+1}=v_{i k}+\Delta t \varphi_{i} S_{i} P_{i k}+\Delta t\left(q_{i n k}^{i}-q_{\text {out }}^{i}\right),
$$

where $v_{i}$ corresponds to the volume in the $i$-th tank at time $k$ (given in cubic meters), $\varphi_{i}$ is the ground absorption coefficient of the $i$-th catchment, $S$ is the surface area, $P_{k}$ is the rain intensity at each sample $k$. Flows $q_{i n}^{i}$ and $q_{\text {out }}{ }_{k}$ are the sum of inflows and outflows, respectively. Real retention tanks, which correspond to the sewer network reservoirs, are modelled in the same way but without the precipitation term. Tanks are connected with flow paths or links, which represent the main sewage pipes between the tanks. Manipulated variables of the system, denoted as $q_{u_{i}}$, are related to the outflows from the control gates. Tank outflows are assumed to be proportional to the tank volume, that is,

$$
q_{\text {out }}^{i} \text { i }=\beta_{i} v_{i k},
$$

where $\beta_{i}$ (given in $\mathrm{s}^{-1}$ ) is defined as the volumelflow conversion (VFC) coefficient as suggested in [12] by using the linear tank model approach. Notice that this relation can be made more accurate (but more complex) if (2) is considered to be non-linear (non-linear tank model approach). Limits on the volume range of real tanks are expressed as

$$
0 \leq v_{i k} \leq \bar{v}_{i}
$$


where $\bar{v}_{i}$ denotes the maximum volume capacity given in cubic meters. As this constraint is physical, it is impossible to send more water to a real tank than it can store. Notice that real tanks without overflow capability have been considered. Virtual tanks do not have a physical upper limit on their capacity. When they rise above a pre-established volume, an overflow situation occurs. This fact represents the case when level in sewers has reached a limit so that an overflow situation can occur in the streets (flooding). Hence, when virtual tanks maximum volume $\bar{v}$ is reached, the exceeded volume above this maximum amount is redirected to another tank (catchment) within the network or to a receiver environment (as pollution). This situation creates a new flow path from the tank denoted as $q_{d}$ (referred to as virtual tank overflow) that can be expressed mathematically as:

$$
q_{d_{k}}= \begin{cases}\frac{\left(v_{k}-\bar{v}\right)}{\Delta t} & \text { if } \quad v_{k} \geq \bar{v} \\ 0 & \text { otherwise. }\end{cases}
$$

In this case, the outflow of virtual tank is then limited by its maximum volume capacity as follows:

$$
q_{\text {out } k}= \begin{cases}\beta \bar{v} & \text { if } \quad v_{k} \geq \bar{v} \\ \beta v_{k} & \text { otherwise. }\end{cases}
$$

Consequently, considering the tank overflow, the difference equation (1) in case of virtual tanks becomes

$$
v_{i k+1}=v_{i k}+\Delta t \varphi_{i} S_{i} P_{i k}+\Delta t\left(q_{i n k}^{i}-q_{\text {out } k}-q_{d k}\right) .
$$

Using the proposed PWLF modelling approach, the tank outflows can be expressed as

$$
\begin{aligned}
q_{\text {out } k} & =\beta \operatorname{sat}\left(v_{k}, \bar{v}\right), \\
q_{d_{k}} & =\frac{\operatorname{dzn}\left(v_{k}, \bar{v}\right)}{\Delta t},
\end{aligned}
$$

On the other hand, as noticed before, real tanks (see Figure 1(b)) are elements designed to retain water in the case of intense rain. For this reason, both tank inflows and outflows are controlled. In the same way, tank inflow is constrained by the current volume within the real tank, by its maximum capacity and by the tank outflow. Since real tanks are considered without overflow capabilities, inflow is premanipulated by using a redirection gate (explained in Section II-B below), what leads to include the management policy in the model of the real tank. Proceeding in this way, the value of the manipulated flow $q_{a k}^{\star}$ is restricted to the maximum flow condition in the input gate, and the flow through input link $q_{a}$ is expressed as

$$
\tilde{q}_{a k}= \begin{cases}q_{a k}^{\star} & \text { if } q_{a k}^{\star} \leq q_{i n k} \\ q_{i n k} & \text { otherwise. }\end{cases}
$$

However, maximum tank capacity also constrains the inflow according to the expression

$$
q_{a k}= \begin{cases}\tilde{q}_{a_{k}} & \text { if } \quad q_{b_{k}}-q_{\text {out }} \leq \frac{\bar{v}-v_{k}}{\Delta t} \\ \frac{\bar{v}-v_{k}}{\Delta t} & \text { otherwise. }\end{cases}
$$

Thus, the real tank outflow is given by

$$
q_{\text {out } k}= \begin{cases}q_{\text {out } k}^{\star} & \text { if } q_{\text {out } k}^{\star} \leq \beta v_{k} \\ \beta v_{k} & \text { otherwise }\end{cases}
$$

taking into account that $q_{\text {out }}^{\star}$ is also limited by the maximum capacity of the outflow link, denoted by $\bar{q}_{\text {out } k}$, leading to the following difference equation:

$$
v_{k+1}=v_{k}+\Delta t\left(q_{a_{k}}-q_{\text {out }}\right) .
$$

Notice that the flow through $q_{b}$ can be derived from the mass balance

$$
q_{b_{k}}=q_{i n_{k}}-q_{a_{k}} .
$$

The PWLF model for this element considering the tank inflow and outflow expressions is

$$
\begin{aligned}
q_{\text {out } k} & =\operatorname{sat}\left(q_{\text {out } k}^{\star}, \beta v_{k}\right) \\
q_{a_{k}} & =\operatorname{sat}\left(q_{a k}^{\star}, \min \left(\frac{\bar{v}-v_{k}}{\Delta t}, q_{\text {in } k}\right)\right) .
\end{aligned}
$$

\section{B. Manipulated Gates}

Within a sewer network, gates are elements used as control devices since they can change the flow downstream. Depending on the action made, gates can be classified as redirection gates, used to change the direction of the sewage flow, and retention gates, used to retain the sewage flow in a certain point (sewer or reservoir) of the network. In the case of real tanks, a retention gate is present to control the outflow. Virtual tank outflows can not be closed but can be diverted using redirection gates. Indeed, redirection gates divert the flow from a nominal path which the flow follows if the gate is closed. This nominal flow is denoted as $Q_{i}$ in the equation below, which expresses the mass conservation relation in the element:

$$
q_{\text {out }}^{i}{ }_{k}^{i}=Q_{i_{k}}+\sum_{j} q_{u_{i} k}^{j}
$$

where $j$ is an index over all manipulated flows coming from the gate. Figure 1(c) shows a conceptual scheme of redirection gates considered in this paper. Assuming that the flow through sewer $q_{a}$ is imposed (for instance computed by means of a control law), the expressions that describe a redirection gate can be written as:

$$
q_{a_{k}}= \begin{cases}\bar{q}_{a} & \text { if } q_{a}^{\star}>\bar{q}_{a} \\ q_{a}^{\star} & \text { otherwise }\end{cases}
$$

where $q_{a}^{\star}$ corresponds to the imposed/computed value for the flow $q_{a k}$. Flow $q_{b_{k}}$ is directly given by the mass balance expression as in (13). In the case of redirection gates, the PWLF model is defined taking into account that $q_{a}$ should satisfy the restriction (17), what can be rewritten in terms of the PWL functions as

$$
q_{a k}=\operatorname{sat}\left(q_{a_{k}}, q_{i n_{k}}\right) \text {. }
$$

Flow through $q_{b}$ is given by the mass balance (13). 


\section{Weirs and Main Sewer Pipes}

Since the description of their dynamics is very close, both are presented together in this section. Nodes are points of the network where the sewage can be either propagated or merged. Hence, these elements can be classified as splitting nodes and merging nodes. The first type can be treated considering a constant partition of the sewage flow in predefined portions according to the topological design characteristics. Merging nodes exhibit a switching behaviour. In the case of a set of $n$ inflows $q_{i}$, with $i=1,2, \ldots, n$, the expression for the node outflow $q_{\text {out }}$ is written as

$$
q_{\text {out }}=\sum_{i=0}^{n} q_{i} .
$$

Weirs can be seen as splitting nodes having a maximum capacity in the nominal outflow path related to the flow capacity of the output pipe. In the same way, main sewer pipes can be seen as weirs with a single inflow. They are used as connection devices between network constitutive elements. Therefore, considering the similarity between all the aforementioned elements and the notation in Figure 1(d), the set of expressions that represent the behaviour either of a weir or a sewer pipe are the following:

$$
\begin{aligned}
& q_{b k}= \begin{cases}\bar{q}_{b} & \text { if } q_{i n}>\bar{q}_{b} \\
q_{i n k} & \text { otherwise, }\end{cases} \\
& q_{c k}= \begin{cases}q_{i n k}-\bar{q}_{b} & \text { if } q_{i n}>\bar{q}_{b} \\
0 & \text { otherwise, }\end{cases}
\end{aligned}
$$

where $\bar{q}_{b}$ is the maximum flow through $q_{b}$ and $q_{i n}$ is the inflow. Notice that the outflow from virtual tanks is assumed to be unlimited in order to guarantee a feasible solution of an associated optimization problem within the design procedure of a optimization-based control strategy. The same idea applies to the outflow $q_{b k}$ related to retention gates. But most often, sewer pipes have limited flow capacity. When the limit of flow capacity is exceeded, the resultant overflow is possibly redirected to another element within the network or is considered as loss to the environment.

The PWLF model for main sewer pipes (or single inflow weirs) can be obtained from the overflow condition as follows:

$$
\begin{aligned}
& q_{b k}=\operatorname{sat}\left(q_{i n_{k}}, \bar{q}_{b}\right), \\
& q_{c k}=\operatorname{dzn}\left(q_{i n_{k}}, \bar{q}_{b}\right),
\end{aligned}
$$

where $\bar{q}_{b}$ corresponds again to the maximum flow capacity of the nominal outflow pipe.

\section{RHC-BASEd RTC ON SEWER Networks}

\section{A. Control Objectives}

The sewage system RHC-control problem has multiple objectives with varying priority, see [4]. The type, number and priority of those objectives can also be different depending on the particular sewage system topology. However, the most common objectives are generally related to the manipulation of the sewage in order to avoid undesired sewage flows outside the main sewers (flooding). The main considered objectives for the case study presented in this paper are listed below in order of decreasing priority:

1) Minimize flooding in streets (virtual tank overflow).

2) Minimize flooding in links between virtual tanks.

3) Maximize sewage treatment.

A secondary purpose of the third objective is to reduce the volume in the tanks to anticipate future rainstorms. This objective also indirectly reduces pollution to the environment. Moreover, this objective can be complemented by conditioning minimum volume in real tanks at the end of the prediction horizon.

\section{B. Problem Constraints}

When using the model representations based on virtual tanks, only flow rates are manipulated in such way that some of the inherent non-linearities (e.g., non-linear relation between gate opening and discharge flow) of the sewer network are simplified as discussed in [3]. But, in turn, some physical restrictions need to be included as constraints on system variables. For instance, variables $q_{u i}^{j}$ that redirect the outflow from a virtual tank should never be larger than the outflow from the tank. This is expressed with the following inequality

$$
\sum_{j} q_{u i k}^{j} \leq q_{\text {out } k}^{i}=\beta v_{i k} .
$$

Additionally, operational constraints associated to the range of gates actuation lead to the manipulated flows have to fulfil $q_{u i k}^{j} \leq \bar{q}_{u i}^{j}$, where $\bar{q}_{u i}^{j}$ denotes the upper limit. Similarly, operational limits on the range of real tank volumes should be included (see (3)) to limit the amount of sewage that can be stored.

\section{RHC disturbances}

Rain plays the role of measured disturbance in the sewer networks RHC problem. The type of disturbance model to be used depends on the rain prediction procedure available. Existing methods range from the use of time series [13] to the sophisticated utilization of meteorological radars [14]. According to [4], different assumptions can be made for the rain prediction when an optimal/predictive control law is used for RTC of sewer networks. Results show that the assumption of constant rain over a short prediction horizon gives results that can be compared with the case of known rain over the considered horizon, confirming similar results that are reported in [3] and [6].

\section{CAse Study Description}

\section{A. The Barcelona Sewer Network}

The city of Barcelona has a CSS of approximately 1697 $\mathrm{km}$ length in the municipal area plus $335 \mathrm{~km}$ in the metropolitan area, but only $514.43 \mathrm{~km}$ are considered as the main sewer network. Its storage capacity is about three million of cubic meters, which implies a dimension three times greater than other cities comparable to Barcelona. It is worth to notice that Barcelona has a population which 
is around 1.59 million inhabitants on a surface of $98 \mathrm{~km}^{2}$, approximately. This fact results in a very high density of population. Additionally, the yearly rainfall is not very high (600 mm/year), but it includes heavy storms (up to $90 \mathrm{~mm} / \mathrm{h}$ ) typical of the Mediterranean climate that can cause a lot of flooding problems and CSO to the receiving environments.

\section{B. Barcelona Test Catchment}

From the whole sewer network of Barcelona, which was described beforehand, this paper considers a portion that includes the main phenomena and the most common characteristics appearing in the entire network. This representative portion is selected since a calibrated and validated model of the network obtainned using the virtual modelling methodology (see Section II) is available as well as rain gauge data for an interval of several years. The considered Barcelona Test Catchment (BTC) has a surface of $22.6 \mathrm{~km}^{2}$ and includes typical elements of the larger network. The fully detailed description of BTC case study including operating ranges of the control signals and state variables as well as the description of all variables and parameters can be found in [8].

Using the virtual tanks representation principle, the resultant BTC model has 12 state variables corresponding to the volumes in the 12 tanks (one real, 11 virtual), four control inputs corresponding to the manipulated links, and five measured disturbances corresponding to the measurements of rain intensity at the sub-catchments. Two WWTPs are used to treat the sewage before it is released to the environment. The states represent the virtual tank volumes are estimated using the limnimeters shown with capital letter $L$ in Figure 2.

The free flows to the environment $\left(q_{10 \mathrm{M}}, q_{7 \mathrm{M}}, q_{8 \mathrm{M}}\right.$ and $q_{11 \mathrm{M}}$ to the Mediterranean sea and $q_{12 s}$ to other catchment) and the flows to the WWTPs $\left(q_{7 \mathrm{~L}}\right.$ and $\left.q_{11 \mathrm{~B}}\right)$ are also shown in Figure 2. The four manipulated links, denoted as $q_{u_{i}}$ have a maximum flow capacity of 9.14, 25, 7 and 29.3 $\mathrm{m}^{3} / \mathrm{s}$, respectively, and these limits can not be relaxed, being physical restrictions of the system (hard constraints).

\section{Simulation And Results}

\section{A. Preliminaries}

This section is focused on comparing the performance of a RHC-based sewer network RTC using a set of real rain episodes when the hybrid modelling approach introduced in [8] and the PWLF-based modelling approach proposed in this paper are considered. Computation time, when every modelling approach is used, is also compared. Computation times presented in this paper has been obtained using Matlab $^{\circledR} 7.2$ implementations running on an Intel ${ }^{\circledR}$ Core $^{\mathrm{TM}} 2$, $2.4 \mathrm{GHz}$ machine with 4Gb RAM. Notice that computation time results reported here related with the use of hybrid models are different with respect to [8] due to the machine characteristics and solver versions.

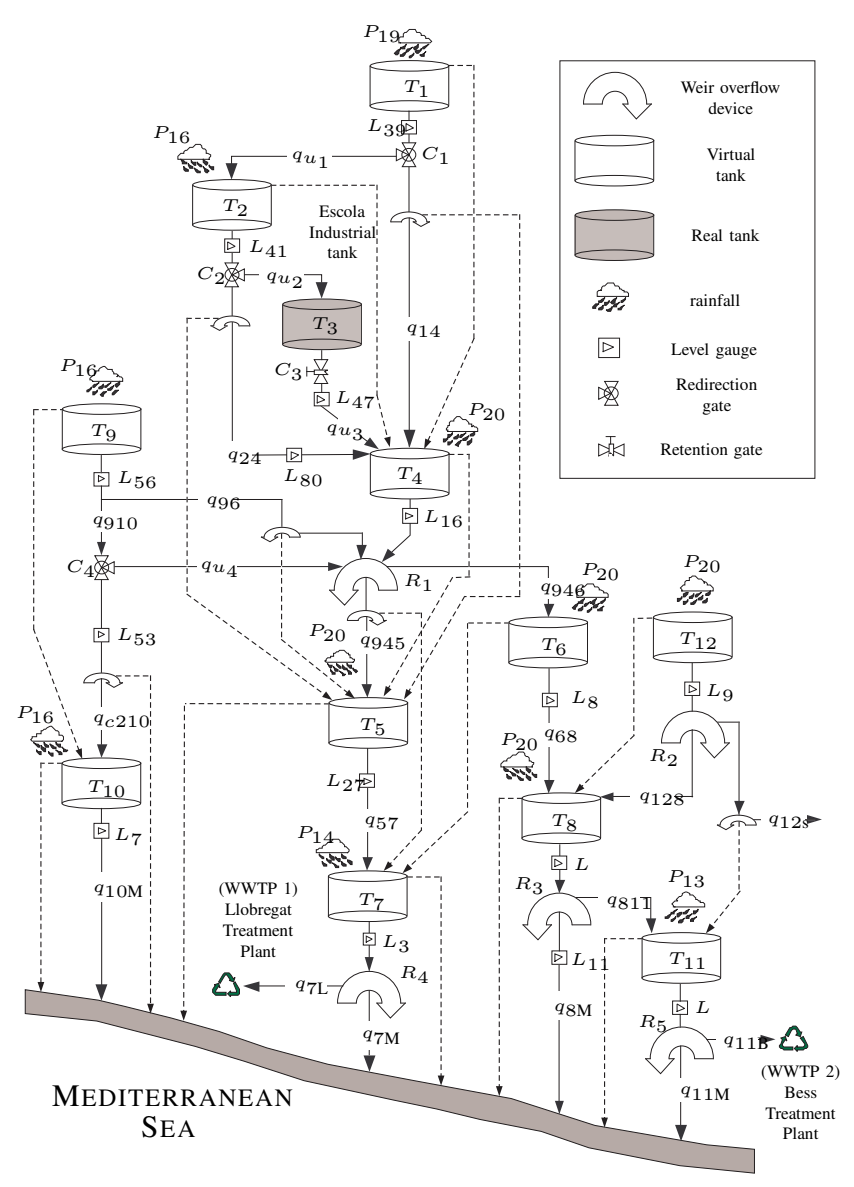

Fig. 2. Barcelona test catchment scheme.

\section{B. Simulation and Prediction Models}

Results presented in this paper are obtained in simulation by using two different models: one used as the plant (sewer network), which in the sequel will be called as openloop model, and the other used by the RHC controller or prediction model. The open-loop model is implemented considering a non-linear representation of the sewer network based on mass balances where ranges and bounds for every variable (control signals, volumes, rain disturbances) are strictly considered and all possible logical or discontinuous dynamics are included (as the case of weirs and overflows). On the other hand, prediction model is obtained by using the modelling approaches compared in this paper. For the comparison, a hybrid RHC controller has been used and the set of considered rain scenarios were simulated using the Hybrid Toolbox for Matlab ${ }^{\circledR}$ (see [15]) and ILOG CPLEX 11.2. This latter solver allows to handle efficiently the mixed-integer programming (MIP) problems associated to the hybrid RHC controller. On the other hand, the model of BTC using the PWLF-based modelling approach was obtained by joining the different compositional elements described in Section II and following the network diagram of Figure 2, resulting in a non-linear representation as a set of expressions for the whole network. The implementation of an RHC using the PWLF-based modelling approach leads to a non-linear 
optimization problem. The selection of the algorithm to solve such problem was done after the evaluation of several solvers available on Tomlab $^{\circledR}$ (e.g., conSolve, nlpSolve, among others). The Structured Trust Region (STR) algorithm (see [16]) was finally chosen because it provides an acceptable tradeoff between the desired system performance and computation time.

\section{RHC Controller Set-up}

According to the discussion in Section III-A to take into account the sewer networks RTC objectives, the following system outputs have been defined in both the hybrid and PWLF-based modelling approaches:

$$
\begin{array}{ll}
y_{1_{k}}=\sum_{i} q_{\operatorname{str}_{v}}+\sum_{j} q_{\operatorname{str}_{q}}, & y_{2_{k}}=\sum_{l} q_{\text {sea }_{k}}, \\
y_{3 k}=q_{\operatorname{trp}_{k}}-\bar{q}_{7 \mathrm{~L}}, & y_{4 k}=q_{\mathrm{trp}_{k}}-\bar{q}_{11 \mathrm{~B}},
\end{array}
$$

where $y_{1 k}$ represents the sum of the $i$ overflows to street from virtual tanks at time $k$, denoted by $q_{\operatorname{str}_{v} k}$, plus the sum of the $j$ overflows to street from links (main pipes) at time $k$, denoted by $q_{\operatorname{str}_{q} k}$. Output $y_{2 k}$ represents the sum of the $l$ overflows which are released to the sea (as receiver environment) at time $k$, denoted as $q_{\text {sea }_{v} k}$, and finally $y_{3 k}$ and $y_{4 k}$ represent the difference at time $k$ between the flows towards the WWTPs, denoted by $q_{\operatorname{trp} 1_{k}}$ and $q_{\operatorname{trp} 2 k}$, and the maximum flow capacity through the associated sewers. For the case study of this paper, $q_{\mathrm{trp}_{k}}=q_{7 \mathrm{~L} k}$ and $q_{\mathrm{trp} 1_{k}}=q_{11 \mathrm{~B} k}$, with their maximum flows $\bar{q}_{7 \mathrm{~L}}$ and $\bar{q}_{11 \mathrm{~B}}$, respectively. Using the outputs (25), the multi-objective cost function for the BTC can be written as follows using the weighted approach technique:

$$
J\left(u_{k}, x_{k}\right)=\sum_{i=1}^{H_{p}}\left\|y_{k+i \mid k}\right\|_{Q}^{2},
$$

where $y_{k+i \mid k}$ is the output vector at the instant $k+i$ with respect to time instant $k$ and $H_{p}$ denotes the prediction horizon, which has been set to 6 (equivalent to 30 minutes) with a sampling time $\Delta t=300 s$. This selection was based on the reaction time of the system to disturbances. Another reason for this selection is that the constant rain prediction assumed in this paper becomes less reliable for larger horizons. The length of the simulation scenarios is 100 samples, what allows to see the influence of the rain peak (disturbance) from the selected rain episode over the dynamics of the network and also over the dynamic of the closed loop. $Q$ corresponds to the weight matrix containning the weights $w_{i}$, each one related to a control objective. Notice that the desired prioritization of the control objectives is given by the values $w_{i}$ that, for this case, determine a $Q$ matrix of the following form $Q=\operatorname{diag}\left\{w_{\text {str }} I \quad w_{\text {sea }} I \quad w_{\text {trp1 }} I \quad w_{\text {trp2 }} I\right\}$, where $I$ corresponds to a identity matrix of suitable dimensions. Here, $w_{\text {str }}=1, w_{\text {sea }}=10^{-1}, w_{\text {trp } 1}=10^{-3}$, and $w_{\text {trp2 }}=10^{-3}$.

Rain episodes used for the simulation of the BTC and for the design of RHC strategies are based on real rain gauge data obtained within the city of Barcelona on the given dates (yyyy-mm-dd in tables) among all five rain gauges for each episode.

\section{Control performance and computation time comparisons}

For the performance comparison purposes, results of the considered indexes when the open-loop scheme is simulated are also presented. The open-loop case consists in the sewage system without control so manipulated links are used as passive elements, i.e., the amount of flows $q_{u 1}, q_{u 2}$ and $q_{u 4}$ only depend on the inflow to the corresponding gate and they are not manipulated while $q_{u 3}$ is the outflow of the real tank given by gravity (tank discharge). Results related to the control performance are summarized in Tables I, II, and III for four representative rain episodes in Barcelona between 1998 and 2002.

TABLE I

Performance Results. Flooding $\left[\times 10^{3} \mathrm{~m}^{3}\right]$

\begin{tabular}{cccc}
\hline Rain Episodes & Open Loop & Hybrid Model & PWLF Model \\
\hline $1999-09-14$ & 108 & 92.9 & 88.2 \\
$2002-10-09$ & 116.1 & 97 & 113.3 \\
$2002-07-31$ & 160.3 & 139.7 & 132.8 \\
$2000-09-28$ & 1 & 1 & 1 \\
\hline
\end{tabular}

TABLE II

Performance Results. Pollution $\left[\times 10^{3} \mathrm{~m}^{3}\right]$

\begin{tabular}{cccc}
\hline Rain Episodes & Open Loop & Hybrid Model & \multicolumn{2}{c}{ PWLF Model } \\
\hline $1999-09-14$ & 225.8 & 223.5 & $226.1(1.16 \%)$ \\
$2002-10-09$ & 409.8 & 398.7 & $407.7(2.25 \%)$ \\
$2002-07-31$ & 378 & 374.6 & $380(1.44 \%)$ \\
$2000-09-28$ & 104.5 & 98 & $102(4.08 \%)$ \\
\hline
\end{tabular}

TABLE III

Performance Results. Treated sewage at WWtPs $\left[\times 10^{3} \mathrm{~m}^{3}\right]$

\begin{tabular}{cccc}
\hline Rain Episodes & Open Loop & Hybrid Model & PWLF Model \\
\hline $1999-09-14$ & 278.3 & 280.7 & $276.7(1.43 \%)$ \\
$2002-10-09$ & 533.8 & 545 & $534.2(1.98 \%)$ \\
$2002-07-31$ & 324.3 & 327.8 & $321.9(1.80 \%)$ \\
$2000-09-28$ & 285.3 & 291.9 & $287.5(1.51 \%)$ \\
\hline
\end{tabular}

Notice from Tables I, II, and III that the system performance is better (according to the given control objectives) when a RHC control law is considered no matter the modelling approach utilized with respect to the performance in open-loop. This justifies the use of closed-loop control. Moreover, the use of the hybrid modelling approach implies in average a better system performance with respect to the performance improvement obtained by using the PWLFbased modelling approach. This performance improvement is basically related to the improvement of the main control objective and then, following in a hierarchical order, to the second objective and so on. In this way, notice that the pollution for some episodes is worse with respect to the open-loop case — see, e.g., Table II, episodes 1999-09-14 and 2002-07-31. However, the performance index associated to the flooding is the best for both episodes, following the pre-established control objectives priority. 
In general, obtained performance results were expected since the RHC controller based on the hybrid modelling approach achieves its optimum by solving a set of convex linear problems using a branch and bound scheme. However, the RHC design using PWLF-based modelling approach leads to a non-linear network model representation what might result in a quasi-convex optimization. Therefore, using the STR algorithm, the global optimum can not be assured because bisection method was not implemented in this paper. This fact leads possibly to a sequence of suboptimal control actions when the computation of the receding horizon control law is done. This explains why the performance obtained using the PWLF model is in general worse than the one obtained using the hybrid model. However, suboptimality levels of the results using the PWLF model were never greater that $4.1 \%$ for the cases of the second and third objective as shown in Tables II, and III in parenthesis at the last column.

TABLE IV

COMPUTATION TIME RESULTS [s]

\begin{tabular}{lcccc}
\hline Rain & \multicolumn{2}{c}{ Hybrid Model } & \multicolumn{2}{c}{ PWLF Model } \\
Episodes & $\begin{array}{c}\text { Total CPU } \\
\text { time }\end{array}$ & $\begin{array}{c}\text { max. CPU } \\
\text { time in } \\
\text { sample }\end{array}$ & $\begin{array}{c}\text { Total CPU } \\
\text { time }\end{array}$ & $\begin{array}{c}\text { max. CPU } \\
\text { time in } \\
\text { sample }\end{array}$ \\
\hline $1999-09-14$ & 1109.29 & 787.17 & 695.33 & 91.32 \\
$2002-10-09$ & 561.73 & 85.31 & 293.23 & 66.01 \\
$2002-07-31$ & 1050.54 & 381.49 & 830.20 & 83.04 \\
$2000-09-28$ & 84.76 & 13.27 & 120.88 & 12.13 \\
\hline
\end{tabular}

On the other hand, the main difference of using the hybrid or the PWLF-based modelling approaches is in the computation time required to determine the control actions at each iteration. As mentioned in Section V-B, the model in MLD form contains an important number of Boolean and auxiliary variables. The complexity of the MIP associated to the RHC law becomes bigger by increasing the number of Boolean variables since the underlying optimization problem is combinatorial and $\mathcal{N} \mathcal{P}$-hard [17]. Thus, the worst-case computation time is exponential in the amount of integer variables. In large-scale systems such as sewer networks, the amount of elements with logical/discontinuous dynamics can augment according to the topology of the particular case study. Therefore, computation times increase towards a point where the use of this modelling for obtaining a RHCbased RTC law becomes almost impossible. Thus, the use of alternative modelling approach based on the PWL functions proposed on this paper allows to have control sequences computed in lower times at the price of some degree of suboptimality due to the possible local optimum. Table IV summarizes the computation times for both the modelling approaches proposed on this paper and for the five rain episodes previously considered. In average, all the maximum computation times to compute the RHC control action when the PWLF modelling approach is used are less than the third part of the sampling time. This is not the case when using the hybrid modelling approach.

\section{Conclusions}

In this paper, receding horizon control of large-scale sewage systems has been addressed considering a modelling approach based on piece-wise linear functions (PWLF). This modelling approach is compared against a hybrid modelling approach previously reported by the authors within the RHC framework. Control performance results and associated computation times of both approaches were compared by using a real case study based on the Barcelona sewer network. With the PWLF-based modelling formulation proposed, although a small amount of suboptimality is introduced since the resultant non-linear optimization problem is non-convex, the reduction in the computation time allows to face the control of large-scale sewer networks. The future work is already focused on the quasi-convexity theoretical proof of optimization problem based on PWLF models in order to take advantage of the modelling using a family of quasiconvex functions.

\section{REFERENCES}

[1] M. Schütze, A. Campisanob, H. Colas, W.Schillingd, and P. Vanrolleghem, "Real time control of urban wastewater systems: Where do we stand today?" Journal of Hydrology, vol. 299, pp. 335-348, 2004.

[2] M. Pleau, H. Colas, P. Lavalle, G. Pelletier, and R. Bonin, "Global optimal real-time control of the Quebec urban drainage system," Environmental Modelling \& Software, vol. 20, pp. 401-413, 2005.

[3] M. Gelormino and N. Ricker, "Model predictive control of a combined sewer system," International Journal of Control, vol. 59, pp. 793-816, 1994.

[4] M. Marinaki and M. Papageorgiou, Optimal Real-time Control of Sewer Networks. Springer, 2005.

[5] S. Duchesne, A. Mailhot, E. Dequidt, and J. Villeneuve, "Mathematical modeling of sewers under surcharge for real time control of combined sewer overflows," Urban Water, vol. 3, pp. 241-252, 2001.

[6] C. Ocampo-Martinez, A. Ingimundarson, V. Puig, and J. Quevedo, "Objective prioritization using lexicographic minimizers for MPC of sewer networks," IEEE Transactions on Control Systems Technology, vol. 16, no. 1, pp. 113-121, 2008.

[7] G. Cembrano, J. Quevedo, M. Salamero, V. Puig, J. Figueras, and J. Mart, "Optimal control of urban drainage systems: a case study," Control Engineering Practice, vol. 12, no. 1, pp. 1-9, 2004.

[8] C. Ocampo-Martinez, A. Bemporad, A. Ingimundarson, and V. Puig, "On hybrid model predictive control of sewer networks," in Identification \& Control: The gap between theory and practice, R. SanchezPeña, V. Puig, and J. Quevedo, Eds. Springer-Verlag, 2007.

[9] A. Bemporad and M. Morari, "Control of systems integrating logic, dynamics, and constraints," Automatica, vol. 35, no. 3, pp. 407-427, 1999.

[10] M. Schechter, "Polyhedral functions and multiparametric linear programming," Journal of Optimization Theory and Applications, vol. 53, pp. 269-280, 1987.

[11] S. Boyd and L. Vandenberghe, Convex Optimization. Cambridge University Press, 2004.

[12] V. Singh, Hydrologic systems: Rainfall-runoff modeling, E. Cliffs, Ed. N.J.: Prentice-Hall, 1988, vol. I.

[13] K. Smith and G. Austin, "Nowcasting precipitation - A proposal for a way forward," Journal of Hydrology, vol. 239, pp. 34-45, 2000.

[14] J. Yuan, K. Tilford, H. Jiang, and I. Cluckie, "Real-time urban drainage system modelling using weather radar rainfall data," Phys. Chem. Earth (B), vol. 24, pp. 915-919, 1999.

[15] A. Bemporad, Hybrid Toolbox - User's Guide, April 2006. [Online]. Available: http://www.dii.unisi.it/hybrid/toolbox

[16] A. R. Conn, N. Gould, A. Sartenaer, and P. L. Toint, "Convergence properties of minimization algorithms for convex constraints using a structured trust region," SIAM Journal on Optimization, vol. 6, no. 4, pp. 1059-1086, 1995.

[17] C. Papadimitriou, Computational Complexity. Addison-Wesley, 1994. 